\title{
White Blood Cells in a Healthy Adolescent Population According to Social and Health Characteristics
}

Isaac Barroso ( $\square$ Isaac.barroso@chsj.min-saude.pt)

Centro Hospitalar de São João EPE: Centro Hospitalar Universitario de Sao Joao https://orcid.org/0000-0002-81634069

Elisabete Ramos

Universidade do Porto Instituto de Saude Publica

Vanda Craveiro

Universidade do Porto Instituto de Saúde Pública: Universidade do Porto Instituto de Saude Publica

João Tiago Guimarães

Universidade do Porto Faculdade de Medicina

\section{Research}

Keywords: Child Health, Immune System, White Blood Cells, Cohort Study

Posted Date: December 28th, 2020

DOI: https://doi.org/10.21203/rs.3.rs-135519/v1

License: (9) This work is licensed under a Creative Commons Attribution 4.0 International License. Read Full License 


\section{Abstract}

Background: The immune system constitutes a sensory system both for intrinsic and extrinsic factors. Among the extrinsic factors, social and environmental determinants of health may influence and shape the immune system from the childhood to adulthood. The aim of this study was to assess the total and differential white blood cells (WBC) according to social and environmental determinants of health in an adolescent population.

Methods: As part of the population-based cohort Epidemiological Health Investigation of Teenagers in Porto (EPITeen), 1213 adolescents were evaluated at the age of 13. Total and differential WBC were evaluated through a venous blood sample. Sociodemographic, behaviour and clinical data were collected by self-administered questionnaires.

Results: Total WBC levels were positively related to female gender, increased body mass index (BMI) and negatively related to private school enrollment, high parental education and practice of sports. The percentage of neutrophils was positively related to female gender, low parental education and lack of sports practice, while the percentage of eosinophils was positively related to chronic disease and medication, allergy, asthma and rhinitis. Monocytes proportion was negatively related to female gender, chronic disease and medication, allergy, asthma and increased BMI.

Conclusions: The presence of disease was associated with an allergic response pattern, while the worse socioeconomic conditions and lack of sports activity were characterized by an inflammatory response pattern. Therefore, our results show that different exposures lead to distinct immune patterns of response that may last and shape the immune system.

\section{Background}

The immune system constitutes a sensory system both for intrinsic and extrinsic factors [1, 2]. Similar to other sensory systems, in order to maintain its responsiveness to changes in stimuli over time, cells of the immune system must adapt to inputs received $[3,4]$. Among the extrinsic factors, social and environmental determinants of health, which includes socioeconomic, demographic, environmental and cultural factors, along with the health system, may influence and shape the immune system from childhood to adulthood [1-3,5-7]. In fact, increasing evidence from epidemiological studies suggest that, in healthy individuals, sociodemographic and health determinants, such as inhaled antigens, infections, vaccinations and behavioural habits do influence and tune the immune system composition and function, particularly the innate component $[1-3,5]$.

These extrinsic factors represent the main cause of the overall interindividual variation in immune cells present in the healthy individuals leading to a lasting impression, although to what extent such exposures can modulate our immune system is yet to be fully disclosed $[2,3,8,9]$. In this regard, the white blood cells (WBC) count is a well-recognized marker of the innate immune response, varying substantially among healthy individuals [10]. While the total and differential WBC have been used to assess the presence of inflammation, several epidemiologic studies have linked elevated WBC counts, with different immune-related diseases, in adults as well as children and adolescents $[10,11]$. In this sense, herein, the main aim of this paper was to study how those variables may affect the innate immune response assessing the total and differential WBC according to several social and environmental determinants in an adolescent population.

\section{Methods}

The participants were adolescents aged 13 years old from the population-based cohort Epidemiological Health Investigation of Teenagers in Porto (EPITeen). As reported elsewhere [12], the EPITeen cohort evaluated adolescents born in 1990, who were enrolled at public and private schools in Porto, Portugal, during the 2003/2004 school year. 


\section{Subjects}

We identified 2786 eligible participants, of whom 2159 agreed to participate and provided information at least for part of the planned assessment, resulting in an overall participation rate of $77.5 \%$, (similar in public (77.7\%) and private (77.0\%) schools ( $p=0.709)$ ). Of the 2159 participants, 772 participants did not perform the blood measurements, 137 had missing data on haematological parameters and 37 presented high-sensitive C-reactive protein ( $h s$-CRP) concentrations $\geq 10 \mathrm{mg} / \mathrm{L}$, which might be indicative of acute infection [13]. Thus, the analysis was based on the information of 1213 participants. Participants' characteristics included in the analysis were compared between non-included participants. Both groups were similar except for type of school (non-included participants were mostly from public schools $81.0 \%$ vs. $72.9 \%, p<0.001)$.

\section{Data collection}

Data were collected using two self-administered questionnaires: one fulfilled at home, comprising information on demographic and social characteristics, family and individual history of disease; another questionnaire was filled at school, which included information on physical activity, smoking and alcohol intake. A physical examination was performed at school, by a trained team, comprising the collection of a blood sample and anthropometric assessment.

\section{Blood measurements}

A venous blood sample was drawn after a 12-hour overnight fast. All the samples were analyzed at the central laboratory of São João Hospital Centre. Total WBC and it's differential percentage (neutrophils, monocytes, lymphocytes, eosinophils and basophils) were obtained using an automated blood counter Sysmex®XE-5000 (Hyogo, Japan). The hsCRP was determined through particle-enhanced immunonephelometry using an auto-analyzer Behring, Nephelometer II (Dade Behring Marburg GMBH, Germany). After excluded those with $h s-C R P \geq 10 \mathrm{mg} / \mathrm{L}$, for the statistical analysis, since $h s$-CRP median was $0.3 \mathrm{mg} / \mathrm{L}$, we dichotomized it as $<0.3 \mathrm{mg} / \mathrm{L}$ and $\geq 0.3 \mathrm{mg} / \mathrm{L}$.

\section{Covariates}

Parental education was obtained by asking the parents the question "What is the highest year of formal schooling you have completed?" and in the analysis it was used the information from the parent with the higher education level. Parental education was then categorized in $\leq 6$ years, 7-9 years, 10-12 years, and $>13$ years of school. Practice of sports was considered the practice of sports in addition to the compulsory school curriculum, regardless of frequency or intensity.

Chronic disease was assessed by asking parents if the child had any illness requiring regular medical care. If the answer was positive, information was gathered on type of disease. The diagnosis of allergy, asthma, rhinitis was collected by asking the parents, separately for each condition, if the child was ever diagnosed with those diseases. Chronic medication was assessed by asking parents if the child had a regular intake of medicines in the previous 12 months. If the answer was positive, information was gathered on type of medication and dosage and frequency/scheme.

Information on vaccination was collected by consulting the vaccination bulletin of each participant, when provided by them $(n=523)$. The assessed vaccines in this study are all part of the Portuguese Childhood Vaccination Schedule except the seasonal flu vaccine [14]. Participants were considered vaccinated if the participants had at least one register in their own bulletin.

Height and weight was measured by a trained team using standardized procedures [12]. Weight and height were obtained with the subject in light indoor clothes and barefoot. Weight was measured in kilograms $(\mathrm{Kg})$, to the $0.1 \mathrm{Kg}$, using a digital scale and height was measured in centimeters $(\mathrm{cm})$, to the nearest $0.1 \mathrm{~cm}$, according to standardized 
procedures. Participants were classified according to the World Health Organization (WHO) sex- and age-specific growth reference [15], as underweight ( $z<-2)$, normal weight $(z \geq-2 \& \leq z+1)$, overweight $(z>+1 \& z \leq+2)$, and obese $(z>+2)$.

\section{Ethical considerations}

The Ethics Committee of the Centro Hospital e Universitário de São João approved this the project, and appropriate standard procedures were developed to guarantee data confidentiality and protection. Written informed consent was obtained from parents and adolescents.

\section{Statistical Analysis}

The distribution of quantitative variables was checked. Results were presented as mean (standard deviation) and compared through the t-test or one-way analysis of variance (ANOVA), when applicable. However, since eosinophils and basophils followed a non-parametric distribution, they were present by median (25th-75th percentiles) and compared through Mann Whitney U test or Kruskal-Wallis, when applicable.

Statistical analyses were performed using the Statistical Package for the Social Sciences (IBM® SPSS Statistics), version 26.0 and statistical significance was considered with an alpha critical value of 0.05 .

\section{Results}

The total WBC levels and their differential percentage according to the participants' sociodemographic and behavioural characteristics are depicted in Table 1. Female participants had significantly higher total WBC levels and percentage of neutrophils, and significantly lower percentage of eosinophils, lymphocytes and monocytes. 
Table 1

Total and differential white blood cells (WBC) according to the participants' sociodemographic and behavioural characteristics.

\begin{tabular}{|c|c|c|c|c|c|c|c|c|}
\hline & & & $\begin{array}{l}\text { WBC } \\
\left(x 10^{9} / L\right)\end{array}$ & $\begin{array}{l}\text { Eosinophils } \\
(\%)\end{array}$ & $\begin{array}{l}\text { Neutrophils } \\
(\%)\end{array}$ & $\begin{array}{l}\text { Lymphocytes } \\
(\%)\end{array}$ & $\begin{array}{l}\text { Monocytes } \\
(\%)\end{array}$ & $\begin{array}{l}\text { Basophils } \\
(\%)\end{array}$ \\
\hline & $\mathbf{n}$ & $\%$ & $\begin{array}{l}\text { Mean } \\
\text { (SD) }\end{array}$ & $\begin{array}{l}\text { Median }\left(P_{25}\right. \\
\left.-P_{75}\right)\end{array}$ & Mean (SD) & Mean (SD) & Mean (SD) & $\begin{array}{l}\text { Median } \\
\left(P_{25}-\right. \\
\left.P_{75}\right)\end{array}$ \\
\hline Overall & & & $\begin{array}{l}6.28 \\
(1.58)\end{array}$ & $\begin{array}{l}2.50(1.50- \\
4.30)\end{array}$ & $\begin{array}{l}52.95 \\
(10.52)\end{array}$ & 35.97 (9.54) & $7.22(2.01)$ & $\begin{array}{l}0.30 \\
(0.20- \\
0.50)\end{array}$ \\
\hline Sex & 1213 & & & & & & & \\
\hline Female & 644 & 53.1 & $\begin{array}{l}6.51 \\
(1.62)\end{array}$ & $\begin{array}{l}1.90(1.23- \\
3.40)\end{array}$ & $\begin{array}{l}55.59 \\
(10.45)\end{array}$ & $34.23(9.57)$ & $6.96(1.98)$ & $\begin{array}{l}0.30 \\
(0.20- \\
0.50)\end{array}$ \\
\hline Male & 569 & 46.9 & $\begin{array}{l}6.02 \\
(1.49)\end{array}$ & $\begin{array}{l}3.10(1.90- \\
5.50)\end{array}$ & $\begin{array}{l}49.96 \\
(9.78)\end{array}$ & $37.94(9.11)$ & $7.52(2.00)$ & $\begin{array}{l}0.30 \\
(0.20- \\
0.50)\end{array}$ \\
\hline p-value & & & $<0.001$ & $<0.001$ & $<0.001$ & $<0.001$ & $<0.001$ & 0.650 \\
\hline $\begin{array}{l}\text { Type of } \\
\text { school }\end{array}$ & 1213 & & & & & & & \\
\hline Public & 884 & 72.9 & $\begin{array}{l}6.35 \\
(1.61)\end{array}$ & $\begin{array}{l}2.55(1.50- \\
4.30)\end{array}$ & $\begin{array}{l}53.03 \\
(10.87)\end{array}$ & 35.77 (9.83) & 7.27 (2.03) & $\begin{array}{l}0.30 \\
(0.20- \\
0.50)\end{array}$ \\
\hline Private & 329 & 27.1 & $\begin{array}{l}6.10 \\
(1.48)\end{array}$ & $\begin{array}{l}2.20(1.30- \\
4.35)\end{array}$ & $\begin{array}{l}52.75 \\
(9.49)\end{array}$ & 36.51 (8.69) & 7.09 (1.97) & $\begin{array}{l}0.40 \\
(0.20- \\
0.50)\end{array}$ \\
\hline p-value & & & 0.013 & 0.041 & 0.667 & 0.203 & 0.165 & 0.007 \\
\hline $\begin{array}{l}\text { Parental } \\
\text { education }\end{array}$ & 1180 & & & & & & & \\
\hline$\leq 6$ years & 301 & 26.3 & $\begin{array}{l}6.61 \\
(1.78)\end{array}$ & $\begin{array}{l}2.40(1.45- \\
4.20)\end{array}$ & $\begin{array}{l}54.58 \\
(10.91)\end{array}$ & $34.36(9.96)$ & 7.27 (1.99) & $\begin{array}{l}0.30 \\
(0.20- \\
0.50)\end{array}$ \\
\hline $7-9$ years & 251 & 21.3 & $\begin{array}{l}6.37 \\
(1.67)\end{array}$ & $\begin{array}{l}2.20(1.40- \\
3.90)\end{array}$ & $\begin{array}{l}54.25 \\
(10.57)\end{array}$ & $35.14(9.85)$ & 7.09 (2.08) & $\begin{array}{l}0.30 \\
(0.20- \\
0.40)\end{array}$ \\
\hline $\begin{array}{l}10- \\
12 \text { years }\end{array}$ & 316 & 26.8 & $\begin{array}{l}6.13 \\
(1.47)\end{array}$ & $\begin{array}{l}2.40(1.40- \\
4.18)\end{array}$ & $\begin{array}{l}52.06 \\
(10.93)\end{array}$ & $36.93(9.64)$ & $7.12(2.01)$ & $\begin{array}{l}0.30 \\
(0.20- \\
0.50)\end{array}$ \\
\hline$\geq 13$ years & 312 & 26.4 & $\begin{array}{l}6.06 \\
(1.35)\end{array}$ & $\begin{array}{l}2.60(1.65- \\
5.10)\end{array}$ & $\begin{array}{l}51.41 \\
(9.42)\end{array}$ & $37.10(8.58)$ & $7.41(2.00)$ & $\begin{array}{l}0.40 \\
(0.20- \\
0.50)\end{array}$ \\
\hline p-value & & & $<0.001$ & 0.140 & $<0.001$ & $<0.001$ & 0.188 & 0.014 \\
\hline $\begin{array}{l}\text { Practice of } \\
\text { sports }\end{array}$ & 1102 & & & & & & & \\
\hline
\end{tabular}




\begin{tabular}{|c|c|c|c|c|c|c|c|c|}
\hline & & & $\begin{array}{l}\text { WBC } \\
\left(x 10^{9} / L\right)\end{array}$ & $\begin{array}{l}\text { Eosinophils } \\
(\%)\end{array}$ & $\begin{array}{l}\text { Neutrophils } \\
(\%)\end{array}$ & $\begin{array}{l}\text { Lymphocytes } \\
\text { (\%) }\end{array}$ & $\begin{array}{l}\text { Monocytes } \\
\text { (\%) }\end{array}$ & $\begin{array}{l}\text { Basophils } \\
(\%)\end{array}$ \\
\hline No & 553 & 50.2 & $\begin{array}{l}6.42 \\
(1.58)\end{array}$ & $\begin{array}{l}2.20(1.30- \\
3.90)\end{array}$ & $\begin{array}{l}54.51 \\
(10.70)\end{array}$ & $34.82(9.73)$ & 7.12 (1.99) & $\begin{array}{l}0.30 \\
(0.20- \\
0.50)\end{array}$ \\
\hline Yes & 549 & 49.8 & $\begin{array}{l}6.18 \\
(1.59)\end{array}$ & $\begin{array}{l}2.70(1.60- \\
4.80)\end{array}$ & $\begin{array}{l}51.71 \\
(10.34)\end{array}$ & $36.82(9.35)$ & 7.33 (2.03) & $\begin{array}{l}0.30 \\
(0.20- \\
0.50)\end{array}$ \\
\hline p-value & & & 0.010 & $<0.001$ & $<0.001$ & 0.001 & 0.079 & 0.169 \\
\hline
\end{tabular}

Participants with better socioeconomic conditions (enrolled at private schools and with higher parental education) showed significantly lower total WBC levels, characterized by lower proportion of neutrophils and higher proportion of lymphocytes. Those who practice sports, presented significantly lower values of total WBC, characterized by significantly higher percentage of eosinophils and lymphocytes and lower neutrophils proportion.

The total WBC levels and their differential percentage according the participants' clinical history are shown in Table 2 . In general, participants with chronic disease, chronic medication or the allergic diseases evaluated were characterized by higher percentage of eosinophils and lower percentage of monocytes. With increasing body mass index (BMI) we found a significant increase on total WBC, characterized by a significantly decreasing proportion of eosinophils and monocytes. Those with higher levels of inflammation, presented higher values of total WBC, with a higher proportion of neutrophils and lower proportion of lymphocytes. Data according the participants' vaccination are drawn in Table 3. In general, no differences were found on total and differential WBC according history of vaccination. Only adolescents who got Haemophilus influenzae type B vaccine had significant lower percentage of basophils, and those who got meningococcal $\mathrm{C}$ vaccine showed significant higher percentage of neutrophils and significant lower percentage of eosinophils and basophils proportion. 
Table 2

Total and differential white blood cells (WBC) according to the participants' clinical history.

\begin{tabular}{|c|c|c|c|c|c|c|c|c|}
\hline & & & $\begin{array}{l}\text { WBC } \\
\left(\times 10^{9} / L\right)\end{array}$ & $\begin{array}{l}\text { Eosinophils } \\
\text { (\%) }\end{array}$ & $\begin{array}{l}\text { Neutrophils } \\
(\%)\end{array}$ & $\begin{array}{l}\text { Lymphocytes } \\
(\%)\end{array}$ & $\begin{array}{l}\text { Monocytes } \\
(\%)\end{array}$ & $\begin{array}{l}\text { Basophils } \\
(\%)\end{array}$ \\
\hline & $\mathrm{n}$ & $\%$ & $\begin{array}{l}\text { Mean } \\
\text { (SD) }\end{array}$ & $\begin{array}{l}\text { Median } \\
\left(P_{25}-P_{75}\right)\end{array}$ & Mean (SD) & Mean (SD) & Mean (SD) & $\begin{array}{l}\text { Median } \\
\left(\mathrm{P}_{25}-\right. \\
\left.\mathrm{P}_{75}\right)\end{array}$ \\
\hline $\begin{array}{l}\text { Chronic } \\
\text { disease }\end{array}$ & 1139 & & & & & & & \\
\hline No & 898 & 78.8 & $\begin{array}{l}6.26 \\
(1.53)\end{array}$ & $\begin{array}{l}2.30(1.48- \\
4.00)\end{array}$ & $\begin{array}{l}53.12 \\
(10.18)\end{array}$ & $36.10(9.50)$ & 7.29 (2.03) & $\begin{array}{l}0.30 \\
(0.20- \\
0.50)\end{array}$ \\
\hline Yes & 241 & 21.2 & $\begin{array}{l}6.37 \\
(1.73)\end{array}$ & $\begin{array}{l}3.10(1.45- \\
6.20)\end{array}$ & $\begin{array}{l}52.58 \\
(11.56)\end{array}$ & 35.47 (9.61) & 6.95 (1.91) & $\begin{array}{l}0.30 \\
(0.20- \\
0.50)\end{array}$ \\
\hline p-value & & & 0.334 & $<0.001$ & 0.510 & 0.365 & 0.021 & 0.916 \\
\hline $\begin{array}{l}\text { Chronic } \\
\text { medication }\end{array}$ & 1108 & & & & & & & \\
\hline No & 488 & 44.0 & $\begin{array}{l}6.32 \\
(1.66)\end{array}$ & $\begin{array}{l}2.20(1.40- \\
4.00)\end{array}$ & $\begin{array}{l}53.72 \\
(11.02)\end{array}$ & $\begin{array}{l}35.36 \\
(10.18)\end{array}$ & 7.39 (2.06) & $\begin{array}{l}0.30 \\
(0.20- \\
0.50)\end{array}$ \\
\hline Yes & 620 & 56.0 & $\begin{array}{l}6.30 \\
(1.55)\end{array}$ & $\begin{array}{l}2.55(1.50- \\
4.70)\end{array}$ & $\begin{array}{l}52.65 \\
(10.19)\end{array}$ & 36.24 (9.01) & 7.06 (1.94) & $\begin{array}{l}0.30 \\
(0.20- \\
0.50)\end{array}$ \\
\hline p-value & & & 0.858 & 0.017 & 0.094 & 0.135 & 0.006 & 0.625 \\
\hline Rhinitis & 1071 & & & & & & & \\
\hline No & 961 & 89.7 & $\begin{array}{l}6.30 \\
(1.61)\end{array}$ & $\begin{array}{l}2.40(1.40- \\
4.00)\end{array}$ & $\begin{array}{l}53.28 \\
(10.66)\end{array}$ & $35.84(9.85)$ & $7.29(2.05)$ & $\begin{array}{l}0.30 \\
(0.20- \\
0.50)\end{array}$ \\
\hline Yes & 110 & 10.3 & $\begin{array}{l}6.25 \\
(1.34)\end{array}$ & $\begin{array}{l}3.50(1.78- \\
6.63)\end{array}$ & $\begin{array}{l}51.39 \\
(10.24)\end{array}$ & $36.16(7.82)$ & $6.85(1.91)$ & $\begin{array}{l}0.40 \\
(0.20- \\
0.50)\end{array}$ \\
\hline$p$-value & & & 0.721 & $<0.001$ & 0.069 & 0.698 & 0.032 & 0.089 \\
\hline Asthma & 1091 & & & & & & & \\
\hline No & 959 & 87.9 & $\begin{array}{l}6.26 \\
(1.59)\end{array}$ & $\begin{array}{l}2.30(1.40- \\
3.80)\end{array}$ & $\begin{array}{l}53.25 \\
(10.61)\end{array}$ & $35.98(9.80)$ & 7.32 (2.07) & $\begin{array}{l}0.30 \\
(0.20- \\
0.50)\end{array}$ \\
\hline Yes & 132 & 12.1 & $\begin{array}{l}6.50 \\
(1.54)\end{array}$ & $\begin{array}{l}4.80(2.43- \\
8.65)\end{array}$ & $\begin{array}{l}51.17 \\
(10.59)\end{array}$ & $35.65(8.41)$ & $6.65(1.66)$ & $\begin{array}{l}0.30 \\
(0.20- \\
0.58)\end{array}$ \\
\hline p-value & & & 0.109 & $<0.001$ & 0.034 & 0.714 & $<0.001$ & 0.194 \\
\hline Allergy & 1093 & & & & & & & \\
\hline No & 797 & 72.9 & $\begin{array}{l}6.25 \\
(1.58)\end{array}$ & $\begin{array}{l}2.30(1.40- \\
3.80)\end{array}$ & $\begin{array}{l}53.20 \\
(10.33)\end{array}$ & 36.16 (9.67) & $7.26(1.96)$ & $\begin{array}{l}0.30 \\
(0.20- \\
0.50)\end{array}$ \\
\hline
\end{tabular}




\begin{tabular}{|c|c|c|c|c|c|c|c|c|}
\hline & & & $\begin{array}{l}\text { WBC } \\
\left(x 10^{9} / L\right)\end{array}$ & $\begin{array}{l}\text { Eosinophils } \\
(\%)\end{array}$ & $\begin{array}{l}\text { Neutrophils } \\
(\%)\end{array}$ & $\begin{array}{l}\text { Lymphocytes } \\
(\%)\end{array}$ & $\begin{array}{l}\text { Monocytes } \\
(\%)\end{array}$ & $\begin{array}{l}\text { Basophils } \\
(\%)\end{array}$ \\
\hline Yes & 296 & 27.1 & $\begin{array}{l}6.43 \\
(1.57)\end{array}$ & $\begin{array}{l}3.10(1.60- \\
6.00)\end{array}$ & $\begin{array}{l}52.83 \\
(11.50)\end{array}$ & 35.19 (9.69) & 7.06 (2.08) & $\begin{array}{l}0.40 \\
(0.20- \\
0.50)\end{array}$ \\
\hline$p$-value & & & 0.105 & $<0.001$ & 0.609 & 0.141 & 0.145 & 0.002 \\
\hline z-scrore BMI & 1210 & & & & & & & \\
\hline $\begin{array}{l}\text { Underweight } \\
(z<-2)\end{array}$ & 13 & 1.1 & $\begin{array}{l}6.34 \\
(1.45)\end{array}$ & $\begin{array}{l}3.50(2.35- \\
7.00)\end{array}$ & $\begin{array}{l}50.09 \\
(10.70)\end{array}$ & $\begin{array}{l}36.53 \\
(10.42)\end{array}$ & $8.03(2.54)$ & $\begin{array}{l}0.40 \\
(0.20- \\
0.65)\end{array}$ \\
\hline $\begin{array}{l}\text { Normal } \\
\text { weight }(z \geq-2 \\
\& \leq z+1)\end{array}$ & 835 & 69.0 & $\begin{array}{l}6.20 \\
(1.62)\end{array}$ & $\begin{array}{l}2.50(1.50- \\
4.60)\end{array}$ & $\begin{array}{l}52.85 \\
(10.44)\end{array}$ & $36.01(9.54)$ & $7.22(2.00)$ & $\begin{array}{l}0.30 \\
(0.20- \\
0.50)\end{array}$ \\
\hline $\begin{array}{l}\text { Overweight (z } \\
>+1 \& z \leq+ \\
\text { 2) }\end{array}$ & 256 & 21.2 & $\begin{array}{l}6.29 \\
(1.45)\end{array}$ & $\begin{array}{l}2.40(1.50- \\
4.00)\end{array}$ & $\begin{array}{l}52.56 \\
(11.31)\end{array}$ & $\begin{array}{l}36.24 \\
(10.33)\end{array}$ & $7.36(2.04)$ & $\begin{array}{l}0.30 \\
(0.20- \\
0.50)\end{array}$ \\
\hline $\begin{array}{l}\text { Obese }(z>+ \\
\text { 2) }\end{array}$ & 106 & 8.8 & $\begin{array}{l}6.74 \\
(1.34)\end{array}$ & $\begin{array}{l}2.20(1.38- \\
3.73)\end{array}$ & $\begin{array}{l}54.75 \\
(8.57)\end{array}$ & $35.27(7.06)$ & 6.79 (1.88) & $\begin{array}{l}0.30 \\
(0.20- \\
0.40)\end{array}$ \\
\hline$p$-value & & & 0.011 & 0.041 & 0.210 & 0.843 & 0.040 & 0.257 \\
\hline hs-CRP & 1213 & & & & & & & \\
\hline$<0.3 \mathrm{mg} / \mathrm{L}$ & 679 & 56.0 & $\begin{array}{l}6.01 \\
(1.35)\end{array}$ & $\begin{array}{l}2.40(1.50- \\
4.20)\end{array}$ & $\begin{array}{l}51.26 \\
(10.24)\end{array}$ & $37.71(9.25)$ & 7.20 (1.96) & $\begin{array}{l}0.30 \\
(0.20- \\
0.50)\end{array}$ \\
\hline$\geq 0.3 \mathrm{mg} / \mathrm{L}$ & 534 & 44.0 & $\begin{array}{l}6.63 \\
(1.77)\end{array}$ & $\begin{array}{l}2.60(1.50- \\
4.45)\end{array}$ & $\begin{array}{l}54.40 \\
(10.54)\end{array}$ & $34.48(9.54)$ & 7.25 (2.07) & $\begin{array}{l}0.30 \\
(0.20- \\
0.50)\end{array}$ \\
\hline$p$-value & & & $<0.001$ & 0.537 & $<0.001$ & $<0.001$ & 0.690 & 0.160 \\
\hline
\end{tabular}


Table 3

Total and differential white blood cells (WBC) according to the participants' vaccination.

\begin{tabular}{|c|c|c|c|c|c|c|c|c|}
\hline & & & $\begin{array}{l}\text { WBC } \\
\left(\times 10^{9} / L\right)\end{array}$ & $\begin{array}{l}\text { Eosinophils } \\
(\%)\end{array}$ & $\begin{array}{l}\text { Neutrophils } \\
(\%)\end{array}$ & $\begin{array}{l}\text { Lymphocytes } \\
(\%)\end{array}$ & $\begin{array}{l}\text { Monocytes } \\
(\%)\end{array}$ & $\begin{array}{l}\text { Basophils } \\
(\%)\end{array}$ \\
\hline & $\mathrm{n}$ & $\%$ & $\begin{array}{l}\text { Mean } \\
\text { (SD) }\end{array}$ & $\begin{array}{l}\text { Median } \\
\left(P_{25}-P_{75}\right)\end{array}$ & Mean (SD) & Mean (SD) & Mean (SD) & $\begin{array}{l}\text { Median } \\
\left(\mathrm{P}_{25}-\right. \\
\left.\mathrm{P}_{75}\right)\end{array}$ \\
\hline $\begin{array}{l}\text { Hepatitis } B \\
\text { virus vaccine }\end{array}$ & 487 & & & & & & & \\
\hline No & 71 & 14.6 & $\begin{array}{l}6.56 \\
(1.93)\end{array}$ & $\begin{array}{l}2.60(1.30- \\
4.80)\end{array}$ & $\begin{array}{l}53.47 \\
(10.08)\end{array}$ & $35.30(9.02)$ & 7.33 (2.13) & $\begin{array}{l}0.40 \\
(0.20- \\
0.50)\end{array}$ \\
\hline Yes & 416 & 85.4 & $\begin{array}{l}6.12 \\
(1.47)\end{array}$ & $\begin{array}{l}2.20(1.50- \\
4.10)\end{array}$ & $\begin{array}{l}52.69 \\
(10.26)\end{array}$ & 36.65 (9.49) & $7.11(1.86)$ & $\begin{array}{l}0.30 \\
(0.20- \\
0.50)\end{array}$ \\
\hline p-value & & & 0.074 & 0.405 & 0.553 & 0.264 & 0.375 & 0.304 \\
\hline $\begin{array}{l}\text { Inactivated } \\
\text { polio vaccine }\end{array}$ & 224 & & & & & & & \\
\hline No & 99 & 44.2 & $\begin{array}{l}6.40 \\
(1.87)\end{array}$ & $\begin{array}{l}2.90(1.60- \\
5.20)\end{array}$ & $\begin{array}{l}52.22 \\
(10.30)\end{array}$ & 35.95 (9.54) & 7.62 (2.23) & $\begin{array}{l}0.40 \\
(0.20- \\
0.60)\end{array}$ \\
\hline Yes & 125 & 55.8 & $\begin{array}{l}6.03 \\
(1.52)\end{array}$ & $\begin{array}{l}2.40(1.50- \\
4.60)\end{array}$ & $\begin{array}{l}52.98 \\
(9.11)\end{array}$ & $35.92(8.63)$ & $7.36(2.02)$ & $\begin{array}{l}0.30 \\
(0.20- \\
0.40)\end{array}$ \\
\hline$p$-value & & & 0.104 & 0.219 & 0.562 & 0.983 & 0.360 & 0.064 \\
\hline $\begin{array}{l}\text { Haemophilus } \\
\text { influenzae type } \\
\text { B vaccine }\end{array}$ & 198 & & & & & & & \\
\hline No & 148 & 74.7 & $\begin{array}{l}6.33 \\
(1.93)\end{array}$ & $\begin{array}{l}2.35(1.60- \\
4.15)\end{array}$ & $\begin{array}{l}52.61 \\
(10.01)\end{array}$ & 36.13 (9.14) & $7.40(2.17)$ & $\begin{array}{l}0.40 \\
(0.20- \\
0.50)\end{array}$ \\
\hline Yes & 50 & 25.3 & $\begin{array}{l}6.47 \\
(1.52)\end{array}$ & $\begin{array}{l}2.15(1.25- \\
4.13)\end{array}$ & $\begin{array}{l}53.84 \\
(9.64)\end{array}$ & 35.49 (8.92) & $7.27(1.56)$ & $\begin{array}{l}0.20 \\
(0.20- \\
0.40)\end{array}$ \\
\hline p-value & & & 0.644 & 0.289 & 0.450 & 0.669 & 0.707 & 0.025 \\
\hline $\begin{array}{l}\text { Meningococcal } \\
\text { C vacccine }\end{array}$ & 330 & & & & & & & \\
\hline No & 114 & 34.5 & $\begin{array}{l}6.25 \\
(1.67)\end{array}$ & $\begin{array}{l}2.90(1.88- \\
4.88)\end{array}$ & $\begin{array}{l}51.03 \\
(9.85)\end{array}$ & 37.02 (8.95) & 7.58 (2.23) & $\begin{array}{l}0.40 \\
(0.20- \\
0.60)\end{array}$ \\
\hline Yes & 216 & 65.5 & $\begin{array}{l}6.13 \\
(1.50)\end{array}$ & $\begin{array}{l}2.00(1.30- \\
4.20)\end{array}$ & $\begin{array}{l}53.95 \\
(9.28)\end{array}$ & $35.26(8.70)$ & $7.20(1.67)$ & $\begin{array}{l}0.30 \\
(0.20- \\
0.50)\end{array}$ \\
\hline$p$-value & & & 0.529 & 0.006 & 0.008 & 0.085 & 0.113 & 0.007 \\
\hline Flu vaccine & 523 & & & & & & & \\
\hline
\end{tabular}




\begin{tabular}{|c|c|c|c|c|c|c|c|c|}
\hline & & & $\begin{array}{l}\text { WBC } \\
\text { (x109/L) }\end{array}$ & $\begin{array}{l}\text { Eosinophils } \\
\text { (\%) }\end{array}$ & $\begin{array}{l}\text { Neutrophils } \\
\text { (\%) }\end{array}$ & $\begin{array}{l}\text { Lymphocytes } \\
\text { (\%) }\end{array}$ & $\begin{array}{l}\text { Monocytes } \\
\text { (\%) }\end{array}$ & $\begin{array}{l}\text { Basophils } \\
\text { (\%) }\end{array}$ \\
\hline No & 386 & 73.8 & $\begin{array}{l}6.25 \\
(1.57)\end{array}$ & $\begin{array}{l}2.30(1.40- \\
4.20)\end{array}$ & $\begin{array}{l}52.85 \\
(10.49)\end{array}$ & $36.33(9.33)$ & $7.10(1.90)$ & $\begin{array}{l}0.30 \\
(0.20- \\
0.50)\end{array}$ \\
\hline Yes & 137 & 26.2 & $\begin{array}{l}6.18 \\
(1.43)\end{array}$ & $\begin{array}{l}2.50(1.40- \\
5.20)\end{array}$ & $\begin{array}{l}51.90 \\
(9.61)\end{array}$ & 36.65 (9.33) & 7.41 (1.92) & $\begin{array}{l}0.30 \\
(0.20- \\
0.50)\end{array}$ \\
\hline$p$-value & & & 0.641 & 0.202 & 0.349 & 0.729 & 0.103 & 0.607 \\
\hline
\end{tabular}

\section{Discussion}

In line with the literature, girls had more total WBC levels and percentage of neutrophils, while boys presented more lymphocytes, monocytes and eosinophils $[11,16]$. Since sex hormones, namely estrogens can be able to shape the immune system, mounting stronger pro-inflammatory responses and leading to an increased immune responsiveness in girls, the higher total WBC levels and neutrophils proportion in females may be explained by sex-differences in this hormones $[4,17]$.

In this study, we reported that a lower parental education and public school enrollment, proxies of low SES, were positively associated with the total WBC levels, as well as a higher proportion of neutrophils and a lower lymphocyte percentage. Actually, lymphocytes, are involved in the suppression of inflammation, representing the regulatory arm of the immune system, while neutrophils promote the increase of systemic inflammation secreting several proinflammatory mediators [18-21]. In addition, a decreased neutrophils-to-lymphocytes ratio (NLR), marker of immune response, was observed for adolescents with higher parental education, as we previously have reported [22]. The pathophysiological mechanism by which socioeconomic status (SES) affects systemic inflammation is still unknown, however, adverse socioeconomic conditions (low SES proxy) and, the consequent exposure to stressful circumstances, could play a significant role in the etiology of inflammation. Actually, recent evidence has shown that a low SES is accompanied by physiological changes in the endocrine and immune systems [23]. In the latter, stress causes leukocytes to become overly aggressive which eventually contributes to increased systemic inflammation [23-26].

Regarding physical activity, in our study active adolescents presented a decreased systemic inflammation with significantly lower total WBC levels and neutrophils percentage and with significantly higher proportion of lymphocytes. Although the underlying mechanisms are not yet fully clarified, Johannsen NM and colleagues stated that physical activity may alter the trafficking of leukocyte subsets between secondary lymphoid organs and blood. These authors also suggest that exercise may lower WBC count through its direct impact on bone marrow haematopoiesis [27]. Furthermore, various studies have shown that increased total WBC levels are related with lower physical activity, albeit most these reports focus on adult subjects [27-30].

Our results are in line with the literature that points asthma and allergy as the most common chronic diseases and medication in adolescence [31, 32]. Actually, for the adolescents diagnosed with allergy, rhinitis and asthma, we found a specific immune response pattern, with higher percentage of eosinophils and basophils as well as lower monocytes proportion. In fact, local eosinophil infiltrate is frequently found in these conditions [33, 34]. Eosinophils activation in the air tract following pharmacological, hormonal, infectious, or environmental stimuli prompts a Th2-driven immune response, which causes airway hyperresponsiveness and chronic remodelling [34]. In consequence, eosinophils are key players in asthma and other allergic diseases by secreting cytokines, chemokines and cytotoxic proteins that contribute to macrophage activation along with mast-cell and basophil degranulation, triggering the release of several allergic mediators $[33,34]$. Furthermore, basophils also promote the progression of the allergic reactions, migrating from the 
blood compartment to inflamed tissues, acting as inflammatory cells [33,35]. On the other hand, regarding the effect of $\mathrm{BMI}$ on the total and differential WBC, we observed an increase in total WBC with the increase of BMI, in accordance with other studies [36-38]. These studies point that changes in the levels of circulating leukocytes may reflect the stimulation of various inflammatory mediators [36-38]. Whilst most of these studies have been conducted in adult populations, Zaldivar and colleagues reported that overweight adolescents had an increase in the circulating leukocytes [39]. The underlying mechanism linking weight gain with an increase in total WBC levels is not fully understood. Nonetheless, leptin may be involved since it stimulates myeloid differentiation, decreasing after weight loss [40, 41].

Our results, globally, showed no significant differences in total and differential WBC between vaccinated and nonvaccinated adolescents. Vaccinations have a substantial impact on the development and shaping of the immune system in childhood, promoting immunological memory and stimulating protective immune responses with a lasting impact on adolescence and adulthood [2, 5, 42]. However, the literature is scarce regarding the long-term effect of the vaccination on the leukocyte count with some studies pointing to no considerable influence on these immune cells, as our results also suggest [43].

This study should be interpreted in light of some limitations. The participants who did not accept perform blood measurements were mostly from public schools representing a potential selection bias, however, in general, there were no significant differences between those included and excluded, indicating that the potential bias is small. Further, the cross-sectional design of this study hinders our ability to infer causality, though it may be fairly helpful for stating future research hypotheses. Finally, the absence of leukocyte immunophenotyping does not allow the evaluation of specific WBC subsets and, therefore, prevents a deeper characterization of the studied population.

On the other hand, the relatively large and apparently healthy sample is one of the strengths of this study since most studies are conducted in small and non-healthy samples. Moreover, since vaccination data was not self-reported but obtained from the vaccination bulletin, greater data reliability and validity was ensured.

\section{Conclusions}

In conclusion, higher levels of WBC were found in the most disadvantaged adolescents, those who do not practice sports, in the obese and in the adolescents who reported a disease condition. However, while the presence of disease was associated with an allergic response pattern (higher percentage of eosinophils and lower monocyte proportion), the worse socioeconomic conditions and absence of sports activity were characterized by an inflammatory response pattern (higher total WBC and percentage of neutrophils as well as lower lymphocytes proportion). Therefore, our results show that different exposures lead to distinct immune patterns of response that may last and shape the immune system. Further prospective studies are warranted to get a better understanding of the associations between immune cells and social and environmental determinants of health that may modulate the immune response, in order to help us to promote long-term immunological health through the optimization of these modifiable determinants.

\section{Abbreviations}

ANOVA - Analysis of Variance

BMI - Body Mass Index

EPITeen - Epidemiological Health Investigation of Teenagers in Porto cohort

hs-CRP - high-sensitive C-reactive Protein

NLR - Neutrophils-to-Lymphocytes Ratio

Page 11/15 
SES - Socioeconomic Status

WBC - White Blood Cells

WHO - World Health Organization

\section{Declarations}

\section{Ethical approval and consent to participate}

The Ethics Committee of the Centro Hospital e Universitário de São João approved this the project, and appropriate standard procedures were developed to guarantee data confidentiality and protection. Written informed consent was obtained from parents and adolescents.

\section{Consent for publication}

Not applicable.

\section{Availability of data and materials}

The datasets used and analysed during the current study are available from the corresponding author on reasonable request.

\section{Competing interests}

The authors declare that they have no competing interests.

\section{Funding}

This study was funded by FEDER through the Operational Programme Competitiveness and Internationalization and national funding from the Foundation for Science and Technology - FCT (Portuguese Ministry of Science, Technology and Higher Education) (POCl-01-0145-FEDER-016829), under Unidade de Investigação em Epidemiologia - Instituto de Saúde Pública da Universidade do Porto (EPIUnit) (P0Cl-01-0145-FEDER-006862; Ref. UID/DTP/04750/2013); and the individual PhD Grant to VC (PD/BD/149264/2019), co-funded by the FCT and the POPH/FSE Program.

\section{Authors' contribution}

I.B: designed the study, collected and analysed the data and wrote the manuscript. V.C: analysed the data. J.T.G and E.R: designed the study and analysed the data.

All authors read and approved the final manuscript.

\section{Acknowledgements}

Not applicable.

\section{References}

1. Olin A, Henckel E, Chen Y, Lakshmikanth T, Pou C, Mikes J, et al. Stereotypic Immune System Development in Newborn Children. Cell 2018; 174(5):1277-92. 
2. MacGillivray DM, Kollmann TR. The role of environmental factors in modulating immune responses in early life. Front Immunol. 2014; 5:434.

3. Brodin P, Jojic V, Gao T, Bhattacharya S, Angel CJ, Furman D, et al. Variation in the human immune system is largely driven by non-heritable influences. Cell. 2015; 160: 37-47.

4. Grossman C. Possible underlying mechanisms of sexual dimorphism in the immune response, fact and hypothesis. Steroid Biochem. 1989; 34(1-6):241-51.

5. Simon AK, Hollander GA, McMichael A. Evolution of the immune system in humans from infancy to old age. Proc Biol Sci. 2015; 282(1821):20143085.

6. Viner RM, Ozer EM, Denny S, Marmot M, Resnick M, Fatusi A, et al. Adolescence and the social determinants of health. Lancet. 2012; 379:1641-52.

7. Mangino M, Roederer M, Beddall M, Nestle FO, Spector TD. Innate and adaptive immune traits are differentially affected by genetic and environmental factors. Nat Commun. 2017; 8:13850.

8. O'Connor MF, Bower JE, Cho HJ, Creswell JD, Dimitrov S, Hamby ME, et al. To assess, to control, to exclude: Effects of biobehavioral factors on circulating inflammatory markers. Brain Behav Immun. 2009; 23(7): 887-97.

9. Kaczorowski KJ, Shekhar K, Nkulikiyimfura D, Dekker CL, Maecker H, Davis MM, et al. Continuous immunotypes describe human immune variation and predict diverse responses. 2017; 114(30): E6097-106.

10. Nalls MA, Couper DJ, Tanaka T, van Rooij FJ, Chen MH, Smith AV, et al. Multiple Loci are associated with white blood cells phenotypes. Plos Genet. 2011; 7(6): e1002113.

11. Pérez-de-Heredia F, Gómez-Martínez S, Díaz LE, Veses AM, Nova E, Wärnberg J, et al. Influence of sex, age, pubertal maturation and body mass index on circulating white blood cell counts in healthy European adolescents-the HELENA study. Eur J Pediatr. 2015; 174(8): 999-1014.

12. Ramos E, Barros H. Family and school determinants of overweight in 13-year-old Portuguese adolescents. Acta Paediatr. 2007; 96(2): 281-6.

13. Yeh E, Willerson J. Coming of age C-reactive protein. Using inflammation markers in cardiology. Circulation. 2003; 107: 370-2.

14. Direccao Geral da Saude. Programa Nacional de Vacinação. https://www.dgs.pt/saude-a-a-z.aspx? $v=\% 3 d \% 3 d B A A A A B \% 2 b L C A A A A A A A B A B L s z U 0 A w A r k 10 a B A A A A A \% 3 d \% 3 d \#$ saude-de-a-a-z/programa-nacional-devacinacao/esquema-recomendado ; 2020 [accessed 10 October 2020].

15. de Onis M, Onyango AW, Borghi E, Siyam A, Nishida C, Siekmann J. Development of a WHO growth reference for school-aged children and adolescents. Bull World Health Organ. 2007; 85(9): 660-7.

16. Romeo J, Wärnberg J, Gómez-Martínez S, Díaz LE, Moreno LA, Castillo MJ, et al. Haematological reference values in Spanish adolescents: the AVENA study. Eur J Haematol. 2009; 83(6):586-94.

17. Verthelyi D. Sex hormones as immunomodulators in health and disease. Int Immunopharmacol. 2001; 1(6):983-93.

18. Grivennikov SI, Greten FR, Karin M. Immunity, inflammation, and cancer. Cell. 2010; 140:883-99.

19. Ait-Oufella H, Salomon BL, Potteaux S, Robertson AKL, Gourdy P, Zoll J, et al. Natural regulatory T cells control the development of atherosclerosis in mice. Nat Med. 2006; 12(2):178-80.

20. Liu X, Zhang Q, Wu H, Du H, Liu L, Shi H, et al. Blood neutrophil to lymphocyte ratio as a predictor of hypertension. Am J Hypertens. 2015; 28:1339-46.

21. Imtiaz F, Shafique K, Mirza SS, Ayoob Z, Vart P, Rao S. Neutrophil lymphocyte ratio as a measure of systemic inflammation in prevalent chronic diseases in Asian population. Int Arch Med. 2012; 5(1):2.

22. Barroso I, Cabral M, Ramos E, Guimaraes JT. Parental education associated with immune function in adolescence. Eur J Public Health. 2020; 30(3):444-48. 
23. Ehrlich KB, Miller GE, Rohleder N, Adam EK. Trajectories of relationship stress and inflammatory processes in adolescence. Dev Psychopathol. 2016; 28(1):127-38.

24. Chiang JJ, Bower JE, Almeida DM, Irwin MR, Seeman TE, Fuligni AJ. Socioeconomic status, daily affective and social experiences, and inflammation during adolescence. Psychosom Med. 2015; 77(3): 256-66.

25. Low CA, Matthews KA, Hall M. Elevated CRP in adolescents: roles of stress and coping. Psychosom Med. 2013; 75:449-52.

26. Risvas G, Papaioannou I, Panagiotakos DB, Farajian P, Bountziouka V, Zampelas A. Perinatal and family factors associated with preadolescence overweight/obesity in Greece: the GRECO study. J Epidemiol Glob Health. 2012; 2(3):145-53.

27. Johannsen NM, Swift DL, Johnson WD, Dixit VD, Earnest CP, Blair SN, et al. Effect of different doses of aerobic exercise on total white blood cell (WBC) and WBC subfraction number in postmenopausal women: results from DREW. PLoS One. 2012; 7(2):e31319.

28. Lee IM, Shiroma EJ, Lobelo F, Puska P, Blair SN, Katzmarzyk PT, et al. Effect of physical inactivity on major noncommunicable diseases worldwide: an analysis of burden of disease and life expectancy. The Lancet. 2012; 380(9838):219-29.

29. Willis EA, Shearer JJ, Matthews CE, Hofmann JN. Association of physical activity and sedentary time with blood cell counts: National Health and Nutrition Survey 2003-2006. PLoS One. 2018; 13(9):e0204277.

30. Pitsavos C, Panagiotakos DB, Chrysohoou C, Kavouras S, Stefanadis C. The associations between physical activity, inflammation, and coagulation markers, in people with metabolic syndrome: the ATTICA study. Eur J Cardiovasc Prev Rehabil. 2005; 12(2):151-58.

31. Wood RA. Asthma, Allergies, and Sinusitis in Adolescence. Adolesc Med. 1997; 8(1):125-38.

32. Sonney J, Insel KC. Exploring the Intersection of Executive Function and Medication Adherence in School-Age Children With Asthma. J Asthma. 2019; 56(2):179-89.

33. Wen T, Rothenberg ME. The Regulatory Function of Eosinophils. Microbiol Spectr. 2016; 4(5):10.

34. Ramirez GA, Yacoub MR, Ripa M, Mannina D, Cariddi A, Saporiti N, et al. Eosinophils from Physiology to Disease: A Comprehensive Review. Biomed Res Int. 2018; 2018:9095275.

35. Chirumbolo S. State-of-the-art review about basophil research in immunology and allergy: is the time right to treat these cells with the respect they deserve? Blood Transfus. 2012; 10(2):148-64.

36. Yoshimura A, Ohnishi S, Orito C, Kawahara Y, Takasaki H, Takeda H, et al. Association of peripheral total and differential leukocyte counts with obesity-related complications in young adults. Obes Facts. 2015; 8(1):1-16.

37. Nieto FJ, Szoklo M, Folsom AR, Rock R, Mercuri M. Leukocyte count correlates in middle-aged adults: the Atherosclerosis Risk in Communities (ARIC) Study. Am J Epidemiol. 1992; 136(5):525-37.

38. Kullo IJ, Hensrud DD, Allison TG. Comparison of numbers of circulating blood monocytes in men grouped by body mass index (<25, 25-<30, $\geq 30)$. Am J Cardiol. 2002; 89:1441-3.

39. Zaldivar F, McMurray RG, Nemet D, Galassetti P, Mills PJ, Cooper DM. Body fat and circulating leukocytes in children. Int J Obes (Lond). 2006; 30(6):906-11.

40. Molina A, Vendrell J, Gutierrez C, Simón I, Masdevall C, Soler J. Insulin resistance, leptin and TNF-alpha system in morbidly obese women after gastric bypass. Obes Surg. 2003; 13:615-21.

41. Veronelli A, Laneri M, Ranieri R, Koprivec D, Vardaro D, Paganelli M, et al. White blood cells in obesity and diabetes: effects of weight loss and normalization of glucose metabolism. Diabetes Care. 2004; 27(10):2501-2.

42. Brodin P, Davis MM. Human immune system variation. Nat Rev Immunol. 2017; 17(1): 21-9. 
43. Kandasamy R, Voysey M, McQuaid F, de Nie K, Ryan R, Orr O, et al. Non-specific immunological effects of selected routine childhood immunisations: systematic review. BMJ. 2016; 355: i5225. 